\title{
Mouvements migratoires entre la Turquie et les Républiques turcophones du Caucase et d'Asie centrale : les impacts religieux
}

\author{
Migrations between Turkey and Turkic-speaking Republics in Caucasus and \\ Central Asia: Religious Impacts \\ Movimientos migratorios entre Turquía y las Repúblicas de lengua turca en el \\ Cáucaso y en Asia central: los impactos religiosos
}

Bayram Balci et Stéphane de Tapia

\section{OpenEdition}

Édition électronique

URL : https://journals.openedition.org/remi/5225

DOI : $10.4000 /$ remi.5225

ISSN : $1777-5418$

Éditeur

Université de Poitiers

Édition imprimée

Date de publication : 1 décembre 2010

Pagination : 133-152

ISBN : 978-2-911627-56-9

ISSN : 0765-0752

\section{Référence électronique}

Bayram Balci et Stéphane de Tapia, « Mouvements migratoires entre la Turquie et les Républiques turcophones du Caucase et d'Asie centrale : les impacts religieux», Revue européenne des migrations internationales [En ligne], vol. 26 - $n^{\circ} 3$ | 2010, mis en ligne le 01 décembre 2013, consulté le 16 avril 2022. URL : http://journals.openedition.org/remi/5225 ; DOI : https://doi.org/10.4000/remi.5225 


\section{Mouvements migratoires entre la Turquie et les Républiques turcophones du Caucase et d'Asie centrale : les impacts religieux}

\section{Bayram BALCI ${ }^{1}$ et Stéphane de TAPIA ${ }^{2}$}

\section{AVANT PROPOS ${ }^{3}$}

T a Turquie et le monde turcophone font partie des régions les mieux couvertes

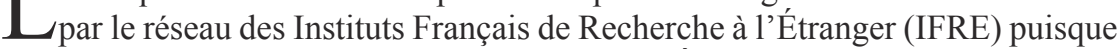
dans ce cadre fonctionnent l'IFEA (Institut Français des Études Anatoliennes) à Istanbul et l'IFEAC (Institut Français d'Études sur l'Asie centrale) à Tachkent, deux instituts qui ont très généreusement contribué à la formation des chercheurs français et plus généralement européens sur le vaste espace turcophone. Peuvent être partiellement adjointes les activités du Centre Franco-russe (Moscou), de l'IFRI (Institut Français de Recherches sur l'Iran, Téhéran), voire de la Délégation Française Archéologique d'Afghanistan (DAFA à Kaboul) puisque les marges de cet espace turcophone ont toujours été en contact avec les mondes russe et iranien étudiés par ces trois centres. La DAFA est bien plus spécialisée sur les périodes anciennes, de l'Antiquité à l'époque médiévale où les incursions turques ont

1 Ingénieur de recherche au CNRS, CERI, 56, rue Jacob, 75006 Paris ; balci_bayram@yahoo.fr

2 Directeur de Recherche au CNRS (Cultures \& Sociétés en Europe) ; stephane.detapia@misha.fr

3 Cet article est issu d'une contribution orale de Bayram Balci au Colloque des IFRE (Institut Français de Recherche à l'Étranger) à Paris et de visites de Stéphane de Tapia en Asie centrale et Azerbaïdjan dans le cadre d'un programme de l'Agence Nationale de la Recherche (Les Suds, aujourd'hui) auquel les deux auteurs collaborent. Intitulé « Caucase et Asie centrale postsoviétique, un autre Sud ? ( SudSov), ce programme cherche à mesurer les évolutions des républiques du sud de l'ancienne URSS et comprend un axe «migrations ». 
joué un grand rôle ${ }^{4}$. Une très grande partie des turcologues, travaillant sur la Turquie ou les républiques turcophones d'Asie centrale, ont effectué des séjours plus ou moins longs dans ces instituts.

Étant nous-mêmes passés par plusieurs de ces IFRE sous des statuts divers ${ }^{5}$, nous proposons aujourd'hui d'observer le phénomène migratoire entre la Turquie et les Républiques turcophones d'Asie centrale et du Caucase, appelé ici « monde turcophone », et d'y analyser plus particulièrement les flux religieux entre ces deux espaces, ou plus exactement comment le phénomène migratoire turc en Asie centrale s'est accompagné de flux religieux qui participent à l'heure actuelle à la recomposition de l'islam dans ces jeunes États. Nous utilisons à la fois les termes «migrants » et " expatriés » turcs sans toujours en montrer la distinction, car la présence turque dans l'ex-URSS est multiforme.

\section{INTRODUCTION}

Un exposé sur la Turquie et le monde turcophone nécessite au préalable une définition de ce "monde turcophone ", appelé aussi " monde turc " ou " turcique ». L'exercice est peu évident tant les concepts utilisés sont flous, polysémiques et tendancieux, puisque certains cachent des sensibilités politiques. Mais partons du constat que depuis le début des années 1990 la Turquie entretient des relations privilégiées avec des États issus de l'ex-URSS et qui partagent avec elle un héritage historico-géographique tant l'Asie centrale est considérée comme la patrie d'origine des Turcs avant leur migration en Anatolie par vagues successives, alors conquêtes effectuées par des nomades pastoraux. En effet, les Empires seldjoukide et ottoman qui verront naître la Turquie contemporaine étaient originaires d'Asie centrale. Ils ont été fondés par des populations nomades projetées depuis leurs steppes centrasiatiques à travers le Khorasan, terme général désignant aux époques antiques et médiévales l'Iran oriental, pour se fixer dans les terres anatoliennes alors byzantines.

Une bonne partie de la population turque actuelle possède des ascendances centrasiatiques, ce qui ne veut nullement dire qu'ethniquement tous les habitants de la Turquie contemporaine sont d'origine centrasiatique. Les populations anatoliennes et proche-anatoliennes déjà sur place ou déportées par les conquérants turco-ottomans ont été mélangées avec les nouveaux venus et constituent la base de la population de la Turquie actuelle. L'histoire plus récente des retraits successifs de l'Empire ottoman sur le déclin a également amené des rapatriés et réfugiés (en turc ottoman muhacir) très nombreux,

4 On parle encore dans les années 1930-1960 d'un Turkestan afghan, car les populations ouzbèkes et turkmènes y sont majoritaires. Historiquement, les dynasties turcophones, comme en Iran, jouent un rôle de premier plan, ne serait-ce que par l'émergence des Ghaznévides au Moyen Âge dont les souverains, anciens esclaves, sont d'origine turque (962-1039).

5 Bayram Balci a été doctorant, pensionnaire à l'IFEAC de 1996 à 1999, correspondant officiel de l'IFEA à Bakou entre 2002 et 2006, puis directeur de l'IFEAC de 2006 à 2010 ; Stéphane de Tapia a fréquenté l'IFEA comme étudiant, puis chercheur CNRS. Il a ensuite visité l'IFEAC et l'antenne IFEA de Bakou comme chercheur et membre de la Commission scientifique du ministère des Affaires étrangères responsable du «pôle TRIAC » (Turquie, Iran, Asie centrale) auquel s'est adjoint récemment le CRF de Moscou. 
originaires des Balkans, du Caucase, de Crimée, de la région Volga-Oural, voire plus récemment encore du Xinjiang et d'Afghanistan, qui ont encore accentué le caractère centrasiatique de la population, peut-être plus sur le plan idéologique que démographique (de Tapia, 1998). Mais malgré ce brassage, la référence en Turquie aux racines centrasiatiques reste constante, et ce malgré le puissant rêve européen qui anime les élites turques comme le reste de la population (Copeaux, 1997, 2000 ; Landau, 1981, 1995a et 1995b).

\section{CONSIDÉRATIONS GÉNÉRALES}

Pour entrer dans le vif du sujet, il s'agit pour nous d'analyser relations et phénomènes migratoires entre la Turquie et plusieurs pays de l'ex-URSS - Azerbaïdjan, Turkménistan, Ouzbékistan, Kirghizstan, Kazakhstan au moins partiellement turcophones -, mais aussi des pays limitrophes comme le Tadjikistan ou des régions autonomes de la Fédération de Russie (comme le Tatarstan), un vaste domaine géographique que les Turcs appellent monde turc (Türk dünyası) désigné en français par l'expression monde « turcophone » ou « turcique » et que la littérature anglo-américaine appelle Turkic ou Turkish World (Çağatay et Kuban, 2006).

La Turquie possède plusieurs points en commun avec ces États : appartenance ethnique, même origine géographique, histoire commune sur plusieurs siècles ${ }^{6}$, religion musulmane principalement sunnite, et surtout langue, même si des différences notables existent entre le turc anatolien et le kazakh, le kirghiz ou l'ouzbek (Bazin, 1986 ; Johanson, 2006). Depuis la fondation de la République turque par Mustafa Kemal en 1923 jusqu'à la dislocation de l'URSS en 1991, le discours en Turquie pour ces populations turciques était très peu élaboré et souvent l'apanage de cercles intellectuels de droite, anti-communistes et aux relents parfois fascisants. Cet intérêt pour les «Turcs de l'Extérieur » (Dlş Türkler) était dans une très large mesure alimenté par des immigrés turciques (Kazakhs, Ouïghours, Ouzbeks, Azéris) qui, fuyant le communisme, avaient trouvé refuge en Turquie, soit directement (Bezanis, 1994), soit indirectement après des passages par la Chine ou l'Afghanistan (Svanberg, 1989a, 1989b ; Salk, 1998). Peu connue en France, cette migration est en réalité très documentée.

À partir de 1990, du fait de la dislocation de l'URSS et de l'avènement sur ces cendres de républiques turciques ou turcophones indépendantes, ce discours à destination des Turcs de l'Extérieur renaît et l'on voit se développer un vaste intérêt pour ces nouveaux " pays frères », oubliés par la majeure partie de la population turque. Un contexte politique favorable (grâce au soutien des pays européens et des États-Unis pour que s'exerce une réelle influence turque dans la région supposée à tort proie facile de l'islamisme et de l'influence iranienne) et des autorités politiques centrasiatiques désemparées (car sommées soudainement de gérer une indépendance quasiment imposée) ont parmi d'autres facteurs

6 La visite de musées historiques à Istanbul, Ankara, Bichkek ou Tachkent, permet de s'en rendre compte, mêmes ancêtres déclarés, mêmes cartes, mêmes références religieuses, objets (archéologie, ethnographie) de factures très proches, mais les chemins se séparent progressivement après l'irruption mongole de l'Empire gengiskhanide et la fracture entre Ottomans sunnites et Séfévides chiites qui partageaient pourtant une origine ethnolinguistique identique, puis plus brutalement avec la prise en main par l’Empire russe puis la soviétisation de 1917 à 1990-91. 
d'ordre géopolitique éveillé en Turquie un rêve de grandeur, celui de constituer et diriger un monde turc uni et solidaire, mais aux contours jamais réellement définis. Ce contexte politique favorable, conjugué à l'esprit d'initiative des jeunes entrepreneurs anatoliens en quête de nouveaux marchés, a poussé des milliers de Turcs vers ces nouvelles républiques. Très rapidement, un vrai phénomène migratoire se développe entre la Turquie et ces républiques, porteur et vecteur de plusieurs discours idéologiques, principalement religieux.

\section{Tentative de description du phénomène migratoire turc en Asie centrale}

Il est difficile d'établir un panorama précis et détaillé, quantitativement et qualitativement du phénomène migratoire anatolien en Asie centrale et dans le Caucase, à savoir principalement en Azerbaïdjan, Turkménistan, Kazakhstan, Ouzbékistan, Kirghizstan. En l'absence de données statistiques quasi inexistantes, ou très parcellaires et souvent sujettes à caution, on ne peut fournir qu'une esquisse générale du phénomène (Balci et Buchwalter, 2001 ; Balci, 2005). Il s’agit généralement de petits commerçants, restaurateurs et responsables de petites et moyennes entreprises, très souvent dans le bâtiment et la construction, mais aussi l'agroalimentaire et la petite industrie (DİYİH passim) ${ }^{7}$. On trouve également des grandes entreprises turques de taille internationale, implantées dans ces pays, dans le domaine de la construction et du textile voire même des hydrocarbures, puisque la compagnie nationale turque de pétrole, TPAO est très active en Azerbaïdjan où elle est associée à la gestion du pétrole de la Caspienne, notamment à son acheminement dans le cadre de l'oléoduc dit BTC Bakou-Tbilissi-Ceyhan (de Tapia, 2008).

Les statistiques font donc défaut sur le nombre exact de migrants turcs dans ce pays, d'abord à cause du manque de services publics, hors Turquie, qui s'intéressent explicitement à cette question, mais aussi du fait qu'un grand nombre de migrants turcs qui travaillent dans ces pays n'ont pas d'enregistrement officiel, et continuent de résider sans aucun titre de séjour légal. Il existe toutefois des estimations qui permettent d'avancer le chiffre de 50000 Turcs en Azerbaïdjan, principalement à Bakou et dans son agglomération proche (Yunusov, 2009). Au Kazakhstan la présence migratoire turque serait beaucoup plus massive, dépassant les 100000 personnes, grâce à une économie locale très dynamique qui attire des travailleurs et des commerçants non seulement dans les deux capitales, Astana et Almaty, mais aussi dans les autres villes du pays, comme Shymkent et Türkistan, où se trouvent des universités turco-kazakhes, mais aussi dans des villes pétrolières comme Atyrau et Aktau sur la Caspienne (DİYİH, passim) ${ }^{8}$. Les nombreuses

7 On peut relever de nombreuses références en turc et en anglais traitant de la présence turque en Asie centrale, rapports officiels (ministères et établissements publics, ambassades, chambres de commerce, etc.) ou articles de chercheurs turcs ayant effectué une partie de leur carrière, stage, recherche, enseignement, dans différentes universités turques. Malheureusement, les données restent partielles, très générales et souvent redondantes.

8 Le rôle des Universités turques mériterait à lui seul une étude appropriée puisqu'elles ont été créées au Turkménistan (Mahtum Kuli), en Kirghizie (Manas et Alatoo), au Kazakhstan (Ahmet Yesevi et Suleyman Demirel), en Azerbaïdjan (Qafqaz). On y enseigne en anglais, russe, turc et langue nationale. Certaines sont liées à l'État turc directement, d'autres, privées au mouvement de Gülen. Les étudiants locaux y apprennent très vite un turc de qualité et ces universités ont généralement une bonne réputation, permettant aussi la projection vers l'Occident. 
entreprises turques importent parfois leur main-d'œuvre adaptée et qualifiée de Turquie, ce qui est parfois source de frictions entre immigrés turcs et travailleurs kazakhs ${ }^{9}$. Au Turkménistan, dont la capitale Achkhabad est en chantier permanent depuis au moins quinze ans (Fenot et Gintrac, 2005), les entreprises turques de BTP, concurrentes directes du groupe français Bouygues, font venir de Turquie des travailleurs turcs dont le nombre global dans le pays avoisine les 20000 , chiffre non négligeable compte tenu de la population du pays qui ne dépasse guère les 4 millions d'habitants. Au Kirghizstan, la présence migratoire turque est tout aussi visible, plus qu'en Ouzbékistan où la nature du régime karimovien (Poujol, 2005 ; Laruelle et Peyrouse, 2006) et les mauvaises relations entre Ankara et Tachkent ont réduit le nombre de Turcs installés en Ouzbékistan ces dernières années.

La migration s'effectue aussi dans l'autre sens, entre républiques turcophones et Turquie. Dans ce sens aussi, l'immigration dite économique liée au commerce et au travail prime. Les chantiers turcs comptent de plus en plus de travailleurs de ces républiques principalement d'Azerbaïdjan et d'Ouzbékistan, mais c'est surtout le commerce qui attire des Centrasiatiques et des Caucasiens en Turquie, notamment le secteur du textile et du cuir. Il faut aussi mentionner l'existence d'une immigration centrasiatique intellectuelle, notamment des chercheurs et des enseignants, des professeurs de musique, et plus largement des comédiens, danseurs et artistes qui viennent faire carrière en Turquie. Enfin, on ne saurait oublier la présence de plusieurs milliers d'étudiants de ces Républiques, inscrits dans les universités turques, avec souvent le soutien financier du gouvernement d'Ankara. Il y aurait à l'heure actuelle entre 2000 et 3000 étudiants de chaque république en Turquie, à l'exception de l'Ouzbékistan qui freine ses relations avec la Turquie.

\section{Les flux idéologiques véhiculés par cette migration à double sens}

Le lien entre migrations, migrants et diffusion d'idées religieuses est une réalité bien connue qui a souvent été étudiée dans les sciences sociales. En cela, notre étude et notre terrain n'ont rien d'original, mais apportent leur pierre à l'édifice général. Ce lien entre migration et circulation d'idées religieuses est par ailleurs complexe, dynamique et interactif. L'immigration peut induire indirectement un message religieux et sa diffusion par la migration, et inversement, le discours religieux peut être la raison même de la migration, c'est-à-dire le résultat de la volonté missionnaire et pèlerine du candidat à la migration. Ne dit-on pas que le Prophète Mohammad, a migré de la Mecque à Médine pour exporter sa foi et la diffuser par la suite dans la péninsule arabique et au-delà, modèle repris par des millions de réfugiés et rapatriés dits muhacir en ottoman, mohajjer dans l'aire irano-pakistanaise ${ }^{10}$. Cette remarque vaut pour toutes les idées, religieuses, mais pas

9 En octobre 2006, un violent incident a opposé travailleurs kazakhs et turcs employés par la firme turque ENKA à la suite de tensions croissantes sur un chantier pétrolier de l'entreprise américaine Chevron. Les Turcs ont compté plus de 150 blessés, dont certains gravement atteints, qu'il a fallu rapatrier en Turquie par avion.

10 De la racine hajr (migration) qui a donné notre Hégire historique, les termes muhacir / mohajjer désignent ces migrants ayant décidé (ou ayant été forcés) de migrer du Dar'ül Harb, terre de conflit, au Dar'ül Islam, terre d'islam. Certains islamistes du «Londonistan » s'autodésignent comme Mohajjirun, les migrants, par référence directe à l'Hégire. 
seulement. Par ailleurs, le message véhiculé peut être en cours de route altéré et prendre des formes inhabituelles, hybrides.

Dans le cas des relations migratoires entre la Turquie et l'Asie centrale, on observe le même phénomène, à savoir que plusieurs courants idéologiques ont été provoqués ou ont provoqué eux-mêmes un mouvement migratoire porteur de messages idéologico-religieux. De fait, le premier discours a été nationaliste et racial, mais n'a pas obtenu tout le résultat escompté (Türk, 2009), ce qui nous permet de l'analyser plus rapidement et de passer à l'examen du principal courant idéologique parti de Turquie, dans les valises des migrants turcs, pour souder ou ressouder cette vaste Asie centrale turcophone à la Turquie et à ses différents courants islamistes.

Jusqu'à la chute de l'Union soviétique, les seuls en Turquie à avoir un discours et un intérêt pour les populations turciques d'URSS étaient les courants nationalistes turquistes, de tendance " droite réactionnaire ». Leur discours prône la grandeur de la race turque et ils vouent un véritable culte au foyer originel des Turcs, l'Asie centrale et la Sibérie, appelé Touran dans la phraséologie nationaliste. Ce courant, au demeurant très hétéroclite et traversé par des clivages de diverses natures, est souvent animé par des réfugiés azéris, kazakhs, ouzbeks, tatars, ouïghours ayant fui le communisme soviétique ou chinois et établis en Turquie où ils créent des associations, des revues et des clubs où la référence à ce Turkestan perdu est constante. Ces courants nostalgiques de la Patrie perdue rêvaient de la dislocation de l'Union soviétique qui permettrait à leur pays de retrouver l'indépendance et la liberté (Landau, 1991 ; Copeaux, 1997).

$\mathrm{Au}$ moment où l'Union soviétique se disloque, ces courants crient victoire et estiment proche le moment où le rêve deviendra réalité avec la création d'un monde turc uni allant de la Turquie jusqu'à la frontière chinoise. Croyant dur comme fer en cette grandeur bientôt retrouvée, certains individus ou courants migrent dans ces pays « enfin libérés du communisme » et en cela ils sont encouragés par un contexte géopolitique international favorable et des autorités politiques qui multiplient les déclarations de retrouvailles entre frères turcs, dans un même monde qui va, selon le président Demirel, «de l'Adriatique à la Muraille de Chine » (Balci, 1999, 2001).

Or, ce rêve s'est vite heurté aux dures réalités du terrain, à savoir la surdité des « frères » turcs à ce discours panturquiste et souvent déconnecté des réalités, bien que des tentatives aient été menées, vite contrées par les gouvernements en place (Türk, 2009). Ces migrants, partis faire du commerce ou des études dans ces pays frères ont constaté avec amertume le décalage entre leurs rêves et la réalité disparate faite de différences de culture, de mentalité et même de langue entre ces peuples pourtant frères. Tirant la conclusion de l'inexistence d'un monde turc, la plupart ont renoncé à leur discours panturquiste et ont bien été obligés d'apprendre le russe, langue de communication par excellence de la région pour pouvoir communiquer avec leurs « frères ».

Une exception mérite tout de même d'être mentionnée et elle concerne l'Azerbaïdjan, où ce discours de retrouvailles entre frères de Turquie et d'Azerbaïdjan n'a pas complètement échoué. Plus que les autres peuples turcophones, les Azéris - la société comme le pouvoir - revendiquent cette fraternité turque avec les autres peuples turco- 
phones, mais surtout avec la Turquie et qui, pour des raisons géopolitiques complexes notamment liées à la question arménienne, ont une vision de la politique régionale convergente avec la Turquie. Et à l'heure actuelle dans les rues de Bakou comme dans les bureaux officiels, on entend très régulièrement, pour qualifier les relations turco azéris, le slogan «Une même Nation, Deux États » (Bir Millet, iki Dövlet). Dans ce cas précis de discours panturquiste entre la Turquie et l'Azerbaïdjan, les migrants en ont été à la fois les porteurs, de même qu'ils ont été portés par ce rêve dans leur choix de migration, même si les considérations économiques ont été aussi très présentes dans le choix et la stratégie migratoires.

\section{LES COURANTS RELIGIEUX AU CENTRE DE CETTE DYNAMIQUE MIGRATOIRE}

Plus que les courants nationalistes, ce sont les mouvements islamistes qui ont le plus bénéficié de l'établissement des liens avec la Turquie. Se sont déversées, dans la vaste Asie Centrale, dans le Caucase et jusque dans les régions musulmanes de la Fédération de Russie, plusieurs vagues successives de missionnaires anatoliens venus islamiser ou réislamiser ces terres fraîchement libérées de l'idéologie communiste et de la propagande soviétique antireligieuse. Pour la plupart ce sont des groupes privés, et en principe indépendants des acteurs étatiques, qui investissent le champ religieux. Mais il convient au préalable d'expliquer l'attitude des États face à l'islam en cette période de fortes turbulences pour tous les régimes issus de l'ex-URSS. Car, même si les flux islamiques entre la Turquie et l'Asie centrale ont été l'œuvre de mouvements privés et fortement liés au phénomène migratoire, les acteurs étatiques n'ont pas été indifférents et leurs perceptions de la situation à l'époque n'ont pas été sans effet sur les résultats des actions missionnaires privées déployées dans ces pays.

Pour la Turquie de ce début des années 1990, comme il a été souligné précédemment, l'espace turcophone du Caucase et d'Asie centrale, représente un nouvel espace d'investissements en termes politiques, économiques et culturels. Outre la mise en place d'un vaste programme éducatif qui permet à des milliers de jeunes étudiants turcs d'étudier en Asie centrale et à des milliers de jeunes centrasiatiques de poursuivre des études en Turquie, l'État turc a aussi, et c'est cela qui est audacieux pour une République laïque et séculière, mis en œuvre un véritable mécanisme de coopération islamique avec ces États, principalement par le biais de ses ambassades où est affecté un attaché aux affaires religieuses chargé d' " exporter » la vision officielle turque de l'islam et de sa place dans la société (Yazgan, 2003). C'est cet attaché qui, par exemple, coordonne les programmes d'échanges entre la Turquie et son pays d'affectation en matière de formation de nouveaux imams et de restauration ou de construction de mosquées. Dans certains cas, il a aussi prise sur les activités religieuses déployées par les migrants turcs dans le pays d'accueil.

Pour les États nouvellement indépendants, la question du contrôle des flux religieux ne se pose pas dans les premières années de leur indépendance. Bien que crispées et anxieuses face à une indépendance davantage imposée par la chute de l'Empire soviétique à partir de Moscou que de la périphérie, les autorités politiques, toutes issues de l'ancien système, se complaisent tout de même dans une euphorie illusoire, celle de l'ouverture vers le monde et de l'établissement de contacts avec des États et de peuples 
longtemps tenus à distance par le pouvoir central soviétique. Dans ce contexte, la circulation des idées et des hommes se développe dans un enthousiasme positif partagé par tous. Des contacts sont établis avec l'extérieur sur la base de solidarités ethniques et/ou religieuses. Un exemple assez révélateur qui relève à la fois de la question migratoire et du phénomène religieux n'est autre que le hadj, le pèlerinage à La Mecque. Très limité pendant la période soviétique, il connaît une forte croissance dès 1992. C'est dans ce cadre bon enfant d'ouverture vers l'extérieur que se développe la rencontre entre idées religieuses en Asie centrale, transportées par des migrants de différentes provenances géographiques, et une population locale avide de contact avec un étranger proche dont le modèle est la Turquie à la fois semblable et différente.

Plusieurs mouvements, organisations, tendances, ou phénomènes confrériques ou néo-confrériques turcs s'intéressent alors à cette région du monde dès cette période où les liens migratoires commencent à s'établir entre la Turquie et l'Asie centrale (Balci, 2003). Nous nous attarderons plus particulièrement sur quatre d'entre eux : le mouvement de Sait Nursî dont les disciples sont appelés Nurcu ; les enseignants éducateurs de Fethullah Gülen qui est un des héritiers spirituels de Sait Nursî ; les disciples de Süleyman Tunahan, une grande figure islamique qui a marqué la Turquie des années 1970 et dont les disciples, nombreux en Asie centrale, sont communément appelés les Süleymancı; des sous-groupes de la grande confrérie nakchibendie qui, à partir de leur base arrière d'Istanbul, ont développé un vaste programme d'assistance humanitaire teinté d'intentions islamiques dans tout l'espace musulman de l'ex-URSS, principalement en Asie centrale. Les frontières entre ces mouvements sont souvent mouvantes et des transfuges peuvent être observés entre groupes, surtout quand les besoins économiques ou les opportunités d'expatriation le rendent nécessaire.

Les Nurcu furent parmi les premiers à saisir l'opportunité offerte par l'ouverture de l'ex-URSS pour s'exporter en Asie centrale. Le terme nurcu renvoie à Sait Nursî, une autorité religieuse née à l'est de la Turquie (1876-1960) et qui occupe une place essentielle dans l'histoire religieuse de la Turquie moderne. Émergeant dans un contexte de fin d'Empire, ce mouvement est avant tout piétiste et cherche à lutter contre la perte de foi et de pratique au sein de la population turque de l'époque, très marquée par les réformes séculières de Kemal Atatürk. Conservateur, mais à la fois nationaliste turc et islamiste modéré, ce mouvement n'est pas politique, mais cherche avant tout à octroyer à l'islam une place centrale au sein de la société turque et dans l'espace public. Interdit et condamné par le régime kémaliste, au lendemain du passage en Turquie à un système politique plus démocratique, notamment dans les années 1950, l'histoire de la confrérie oscille entre bannissement et existence légale. À la mort du père fondateur, le mouvement se scinde en plusieurs tendances, mais toutes sont caractérisées par une pratique militante qui cherche à desserrer le contrôle excessif de l'État sur la religion en Turquie.

Au début des années 1990, différentes tendances issues de ce mouvement s'exportent en Asie centrale, très souvent par le biais du petit commerce et du travail dans les grandes entreprises turques de construction. Le discours religieux des membres de ces différentes associations ou mouvances spirituelles marquées par l'héritage de Nursî n'a rien de révolutionnaire. La plupart du temps leur action se limite à organiser, dans des madrasas semi-officielles ou simplement dans des maisons ou appartements collectifs, des 
lectures de l'œuvre maîtresse de leur père spirituel, la Risale-i Nur. Ce texte, «Épître de la Lumière ", n'est autre qu'une exégèse du Coran, écrite dans une langue plus ottomane que turque, à tel point que sans un dictionnaire ou l'assistance d'un maître, sa lecture ou sa compréhension s'en trouve quasiment contrariées. Semi-légales, les activités de ces migrants turcs sont selon les pays sous étroite surveillance. Le mouvement comptait énormément sur les possibilités d'action prosélyte en Ouzbékistan, pays le plus peuplé et le plus musulman de la région. Or, les relations diplomatiques tendues entre Ankara et Tachkent, et surtout le manque flagrant de libertés religieuses ont considérablement freiné son implantation dans le pays.

Issu de ce même mouvement fondé par Sait Nursî, l'organisation de Fethullah Gülen, un gourou à la mode anatolienne, mérite une place particulière dans l'analyse tant son action et son impact sont considérables dans l'exportation d'un islam turc en Asie centrale et dans le Caucase. Le mouvement, doté d'une identité à géométrie variable, tantôt néo-confrérie, tantôt mouvance ou même nébuleuse est fortement lié à la personnalité de son fondateur Fethullah Gülen, un des nombreux héritiers de Sait Nursî. Aux contours imprécis, cette mouvance religieuse s'est formée sur une longue période, entre les années 1970 où, dans la région d'Izmir, Gülen et ses proches créent plusieurs associations culturelles et éducatives, et les années 1990, décennie caractérisée en Turquie par une remarquable entrée dans la libéralisation économique permettant l'émergence de centaines d'entreprises économiques très fortement structurées autour de valeurs islamiques. À partir d'un noyau d'associations rassemblant des parents d'élèves et des éducateurs, la mouvance gagne la confiance de milliers d'entreprises et d'entrepreneurs et parvient à bâtir un pseudo « holding » sans personnalité juridique distincte et précise, mais disposant d'un puissant levier économique et financier dépassant les frontières de la Turquie.

Le contenu idéologique de la tendance est difficilement identifiable, car Fethullah Gülen se veut apolitique et ses prédications et discours empruntent à des registres très variés : mystique soufie, nationalisme turc, islam progressiste et moderniste, œcuménisme islamo-chrétien entre autres. À la fin des années 1980, le mouvement est à son apogée et commence même à inquiéter les gardiens du dogme kémaliste, si bien que l'Armée et les milieux séculiers craignent sa possible transformation en force politique, après une longue période de gestation dans un cadre éducatif et associatif. Cette fin de décennie est aussi celle de l'établissement de liens divers entre la Turquie et l'ex-URSS, une opportunité, une aubaine pour les entreprises proches de Gülen désormais trop puissantes pour se limiter au territoire proprement turc et à la recherche de nouveaux marchés commerciaux et de nouvelles terres de prédication.

C'est ainsi que des petits commerçants, des travailleurs, des éducateurs et parfois même des entreprises de taille internationale, se trouvant plus ou moins dans la sphère spirituelle de Fethullah Gülen, se sont exportés en Asie centrale pour y travailler et propager par la même occasion les idées de leur maître. Ces disciples de Gülen, les fethullahçı, s'illustrent notamment par la création et l'animation de tout un réseau éducatif de lycées et des centres de langue, voire même des centres de préparation aux concours universitaires. L'enseignement y est séculier et laïc en toute conformité aux normes nationales héritées de la période soviétique ; aucun enseignement coranique ou islamique n'est 
dispensé dans les lycées et aucune littérature islamique n'y est propagée. Mais, et c'est là toute la subtilité de leur méthode, sans cours de religion dans des écoles prétendument laïques, le mouvement parvient tout de même à véhiculer la pensée de Fethullah Gülen, prônant un islam à la fois turc national, en bons termes avec l'État en place, marqué par le soufisme et dépourvu de toute ambition politique. Dans chaque pays d'Asie centrale, les dizaines d'écoles fondées par le mouvement travaillent en symbiose avec des entreprises turques implantées dans le pays (Balci, 2003). Le corps enseignant est constitué de jeunes professeurs, tout juste sortis des universités turques, partis en Asie centrale pour des motivations à la fois économiques et spirituelles, et pour diffuser une forme évoluée d'islam dans une région considérée comme le pays d'origine des ancêtres. Comparables, toute proportion gardée, aux jésuites partis d'Europe occidentale pour propager la bonne parole chrétienne à travers le monde, les cadres supérieurs de Gülen avouent volontiers s'inspirer de ce phénomène missionnaire pour, à leur tour, exporter leur foi et leur culture dans de nouvelles contrées.

Un autre mouvement turc, moins structuré et moins riche, mais avec la même logique de dissémination de migrants anatoliens en Asie centrale et dans le Caucase, tente à sa manière de contribuer au flux spirituel qui à partir de la Turquie vient irriguer les friches postsoviétiques. Il s'agit des disciples d'un autre grand intellectuel musulman turc, Hilmi Süleyman Tunahan, appelés les Süleymancı, qui diffusent, plus ouvertement que les Fethullahçı, la philosophie islamique de leur maître. Le mouvement en Turquie du temps de son fondateur s'était fait remarquer par ses querelles constantes avec la Diyanet, Présidence des affaires religieuses sous tutelle du Premier ministre, la plus haute instance officielle de gestion de l'islam turc, qui voulait imposer son monopole sur la gestion de l'islam en Turquie, ce que refusaient certains mouvements privés dont celui de Süleyman Tunahan. Le mot d'ordre du mouvement a toujours été et demeure " permettre à chaque musulman de pouvoir lire le Coran en arabe ». Leitmotiv qui n'a guère changé puisqu'en Turquie comme dans leurs pays d'expatriation les militants du mouvement placent au centre de leurs activités et de leurs préoccupations la fondation d'écoles coraniques, où la priorité absolue est de permettre aux jeunes générations l'accès au Coran en langue arabe. L'islam promu par ces expatriés turcs véhicule aussi une certaine idéologie turquiste (Bozay, 2005), du moins promeut une image positive de la Turquie (Ateş, Karakaş et Ortayl1, 2005). Dans chaque pays où le mouvement est implanté, il entretient de bonnes relations avec les instances officielles de l'islam et les organisations islamiques modérées.

Autant que les autres mouvements turcs, cette mouvance s'appuie sur les commerçants et petits entrepreneurs turcs pour se développer dans ces pays. Elle a ouvert dans tous les pays de la région, sauf en Ouzbékistan et au Tadjikistan, de modestes madrasas entièrement financées par des fondations et associations implantées en Turquie et des entreprises turques présentes en Asie centrale et dans le Caucase. Régulièrement des nouveaux cadres religieux arrivent de Turquie et certains jeunes étudiants sont envoyés dans les fondations privées Süleymancı partout en Turquie pour y perfectionner leur formation. Cette mouvance s'est surtout implantée dans la région caucasienne, en Azerbaïdjan et en Géorgie, notamment dans la province d'Adjarie. Particularité de leur présence dans cette région, la mouvance utilise les descendants de la diaspora caucasienne en Turquie pour diffuser leurs idées. En effet, il existe en Turquie de très nombreuses associations de descendants des mouvements diasporiques caucasiens, qui avaient trouvé refuge en 
Turquie ottomane pour fuir la domination russe. Depuis la fin de l'URSS, une partie des échanges, de tout genre, entre la Turquie et le Caucase est le fait de ces enfants de la diaspora. Ainsi, une bonne partie des missionnaires envoyés dans ces contrées ont des origines caucasiennes.

Les Nakchibendis constituent le quatrième et dernier groupe turc important à s'être beaucoup intéressé à l'Asie centrale. Ainsi des organisations nakchibendies ont développé, en s'appuyant sur nombre de volontaires turcs au départ, plusieurs activités pour renouer le contact avec les nakchibendis de l'ex-URSS et pour apporter leur contribution au processus de réislamisation de cette région. La nakchibendiyya, du nom de Bahaduddin Nakchibendî, un mystique du XVe siècle né à dix kilomètres de Boukhara, est une confrérie soufie qui à partir de ce foyer centrasiatique s'est diffusée dans tout le monde musulman, principalement en Inde, dans le Caucase et en Turquie. Prônant un islam mystique modéré, mais plus ou moins politisé selon les époques et les lieux, cette confrérie occupe une place essentielle dans le champ islamique de Turquie où elle est incarnée par plusieurs branches animées par d'importantes autorités religieuses. Dans leur ensemble, quelque soit leur branche, les nakchibendis de Turquie ont toujours entretenu une forte nostalgie pour la patrie du fondateur de l'ordre et l'on sait qu'au début des années 1990, Turgut Ozal, Premier ministre et président de la République par la suite, par admiration pour cet ordre qui lui avait apporté un soutien politique et électoraliste non négligeable, a généreusement contribué à la restauration du mausolée et facilité les premiers contacts entre Nakchibendis de Turquie et leurs confrères d'Ouzbékistan.

Forts de ces soutiens, les disciples de cette confrérie ont également utilisé le flux important des travailleurs turcs en Asie centrale pour mettre leur entreprise mystique à l'épreuve du contact avec la patrie de la nakchibendiyya. Mais surtout, dans le cas de cette confrérie, c'est davantage le pèlerinage sur la tombe de Bahauddin Nakchibend à Boukhara qui a permis de recréer des liens entre ses disciples de Turquie, d'Asie centrale et d'ailleurs. Un véritable tourisme religieux, impensable pendant la période soviétique, s'est développé à partir de la Turquie d'où des compagnies proposaient dès 1990 un parcours incluant Tachkent, Boukhara, Ghijduvan et Samarkand, où se trouvent d'importants sites liés à la nakchibendiyya ou à d'autres ordres, comme la yasaviyya dont le berceau se trouve dans la ville aujourd'hui kazakhe de Turkestan (Balci, 2001).

Paradoxalement, c'est moins dans la patrie originelle de la nakchibendiyya, en Asie centrale, que dans les villes du Caucase, en Azerbaïdjan et en Géorgie notamment, que les nakchibendis turcs ont été les plus influents et les plus à même de proposer leurs " services ", à savoir un enseignement islamique en langue turque des œuvres caritatives, la restauration ou reconstruction de certaines mosquées ayant souffert par le manque d'entretien pendant la période soviétique, etc. En Azerbaïdjan, plus massivement dans les régions sunnites que chiites, la branche nakchibendie, liée à la fondation Mahmut Hudayi et animée à l'heure actuelle par le leader nakchibendis Osman Nuri Topbaş et originaire de la ville d'Üsküdar, dans l'agglomération d'Istanbul, gère une demi-douzaine d'établissements religieux dans lesquels se forment les nouvelles élites musulmanes du pays (Balci et Motika, 2007). Comme dans le cas des autres mouvements, d'importants échanges et de va-et-vient ont lieu entre la Turquie, et le pays d'implantation, pour permettre le transfert de savoir dans les deux sens. Car si les Turcs cherchent à exporter leur vision de l'islam, ils 
reçoivent en retour, à un moindre degré certes, des influences islamiques issues des traditions de ces pays-là et plus particulièrement d'Asie centrale d'où sont originaires nombre d'étudiants venus en Turquie pour leurs études universitaires.

\section{Comment mesurer l'impact de ces flux religieux sur les sociétés d'Asie centrale et du Caucase ?}

Bien que déployé sur une courte période, de 1991 à nos jours, le phénomène migratoire en Asie centrale et dans le Caucase a particulièrement affecté l'espace urbain et les sociétés locales. La présence turque expatriée ou immigrée, dans la sphère économique, industrielle, commerciale ou bien culturelle, éducative et religieuse est particulièrement visible et fait partie des réalités quotidiennes des grandes villes de Bakou à Almaty en passant par Achkhabad, Tachkent, Bichkek et Astana (Güler, 2007) ${ }^{11}$. La croissance des mariages mixtes favorise un certain brassage et accentue le phénomène migratoire définitif de ceux qui au départ n'étaient qu'expatriés. Même si en termes de gros capitaux la présence économique turque est plus faible que celle des grandes puissances économiques mondiales comme l'Allemagne ou les États-Unis, les entreprises étrangères de taille moyenne sont très souvent originaires de Turquie. Sans même que l'enseignement du turc soit particulièrement présent dans les écoles et les universités, le turc anatolien est connu voire pratiqué par de plus en plus de gens, grâce à la forte et constante présence turque depuis plus de quinze ans. La proximité linguistique entre le turc anatolien et les langues turciques locales facilite aux Turcs l'apprentissage de l'ouzbek, du kazakh mais inconsciemment ou consciemment c'est le turc qu'ils imposent à leurs partenaires locaux. Dans les grandes entreprises turques en tout cas, la langue de travail est le turc, devant la langue officielle locale (kazakhe, ouzbek, kirghize, turkmène, etc.) et le russe.

En termes religieux, l'impact de la migration turque en Asie centrale est réel, bien que difficile à évaluer avec précision, et est souvent tributaire de la nature des relations entre la Turquie et chacun des États de la région. De même, le degré de liberté qui y prévaut, notamment en matière de liberté religieuse, détermine le degré d'impact des missionnaires turcs en Asie centrale.

Dans un souci de simplicité, procédons à un bilan par pays, et commençons par l'État le plus proche, à tous points de vue, de la Turquie, l'Azerbaïdjan où les migrants turcs ont été parmi les premiers à s'implanter dès 1990, dans certaines villes avant même les religieux iraniens pourtant très proches de ce pays (Balci et Motika, 2007). En convergence avec l'action de l'État turc qui leur a facilité la tâche, et pour une série de raisons à la fois politiques et géopolitiques (la question arménienne notamment), les migrants turcs affiliés à l'une ou l'autre des organisations religieuses évoquées ici ont déjà formé des centaines de membres des nouvelles élites religieuses à travers tout le pays. Au sein même de l'appareil de gestion de l'islam, la direction des affaires religieuses, mais aussi le comité d'État pour les affaires religieuses, on trouve de nombreux cadres formés sur place par les fondations caritatives turques. En cela, les organismes privés, originaires d'un pays

11 Écrit par un cadre d'entreprise privée qui a effectué une partie non négligeable de sa carrière dans la région, cet ouvrage est un plaidoyer pour une approche plus pragmatique et plus réaliste des sociétés locales par les entrepreneurs et surtout les pouvoirs publics turcs.

REMI 2010 (26) 3 pp. 133-152 
laïc et séculier comme la Turquie, ont été plus performants que les missionnaires envoyés par la République islamique d'Iran. Cette plus grande performance des mouvements turcs montre à quel point le contexte politique est important, y compris quand il s'agit de la circulation des idées religieuses.

Le Turkménistan est aussi un cas assez intéressant dans cette analyse des flux religieux turcs véhiculés en Asie centrale. Malgré la fermeture de son régime et son manque de libertés religieuses, le Turkménistan a été un terrain de prédication particulièrement bien investi par les mouvements islamiques turcs, mais aussi par l'État turc dont l'attaché aux affaires religieuses près l'ambassade de Turquie gère depuis plus de quinze ans de vrais programmes de coopération islamique, notamment en termes de formation grâce à la création d'une faculté de théologie. Une visite dans la plupart des villes de ce pays, avec à chaque fois des haltes importantes dans les principales mosquées, nous a permis de mesurer l'importance de cet impact turc sur l'islam turkmène. La plupart des imams formés depuis l'indépendance du pays, ont un rapport direct avec la Turquie : ils ont été formés soit par l'État turc, soit par des fondations privées qui déploient leurs activités dans le pays d'une manière pas toujours légale d'ailleurs.

L'Ouzbékistan est un cas plus complexe à analyser, tant les relations avec la Turquie se sont dégradées depuis le milieu des années 1990, après avoir été si chaleureuses et cordiales durant les premières années de l'indépendance ouzbèke. Pour les résumer à l'extrême, les relations ouzbéko-turques ont souffert du fait que l'opposition au régime de Tachkent, les partis Erk et Birlik, se sont installés dès 1994 en Turquie où ils ont bénéficié $\mathrm{du}$ soutien de certains milieux nationalistes turcs, sans parvenir toutefois à gagner la confiance des autorités officielles turques (Türk, 2009). En revanche, ils étaient particulièrement populaires parmi les milliers d'étudiants ouzbeks envoyés par Tachkent en Turquie, si bien que rapidement cette opposition ouzbèke a inquiété le régime de Karimov qui, de tout temps suspicieux à l'égard de toute idée subversive, a fait rapatrier la quasitotalité des étudiants ouzbeks de Turquie et gelé ses relations avec la Turquie.

En dépit de la méfiance des Ouzbèks qui ont toujours étroitement surveillé toute influence extérieure, certains mouvements islamiques turcs se sont bien implantés dans les principales villes ouzbèkes tout au long des années 1990. Le mouvement de Gülen était présent massivement dans tout le pays, mais à partir de septembre 2001, le pays, sombrant dans la paranoïa a mené une véritable chasse aux sorcières contre les Turcs, accusés de subversion. Toutes les écoles ont été fermées du jour au lendemain ; certains entrepreneurs liés à ces écoles ont été expulsés, et des Ouzbeks compromis avec ces établissements ont écopé de peines de prison. Cette épuration n'a pas complètement réussi à éradiquer les idées déjà véhiculées par ces missionnaires turcs puisqu'en avril 2009 plusieurs membres ouzbeks soupçonnés d'appartenir à la mouvance nurcu et de propager leurs idées subversives ont été arrêtés et mis en prison. 
Le Kirghizstan, mais surtout le Kazakhstan avec ses pharaoniques projets de construction dopés par la manne pétrolière, attire de nombreux entrepreneurs étrangers ${ }^{12}$. Parmi eux, des Turcs originaires de la Turquie profonde, de cette Anatolie moralement conservatrice, mais économiquement très dynamique. Les mouvements islamistes turcs évoqués, Nurcu, Fethullahçı et autres, trouvent un terrain particulièrement propice à leurs activités. Ils sont en cela indirectement encouragés par les autorités kazakhes qui y voient un moyen de favoriser le renouveau de la culture kazakhe dont les Turcs se font les porte-parole sur place, dans un contexte démographique où l'élément slave est encore très important puisqu'environ $40 \%$ de la population est russe ou russophone. Pour les autorités kazakhes, la présence turque fait contrepoids à la forte composante slave de la population. Pour les missionnaires turcs, avec une université à Almaty, trente-cinq établissements de niveau lycée répartis dans tout le pays, et une vingtaine de modestes madrasas gérées par les Süleymancl, le Kazakhstan est une terre de conquête, un vaste territoire vierge, peuplé d'autant de Russes que de Kazakhs, tous très perméables à la propagande religieuse qui y faisait défaut depuis longtemps. Aujourd'hui, au Kirghizstan comme au Kazakhstan, tous les cadres religieux sont redevables d'une manière ou d'une autre aux autorités religieuses turques ou à ces mouvements religieux turcs confrériques privés. Tant et si bien que naturellement l'islam turc sous toutes ses formes exerce une influence considérable sur l'establishment religieux local, voire même sur les nouvelles élites politiques souvent tentées de s'inspirer de la Turquie pour bâtir une nouvelle nation où le facteur religieux n'est pas totalement marginalisé.

L'islam ou plutôt les islams de Turquie ont une aura bien plus large et leur influence dépasse le cadre des républiques turcophones puisque le Tadjikistan, les régions turcophones de la Fédération de Russie, notamment le Tatarstan et le Bachkortostan, sont également des terrains importants pour leur action missionnaire appuyée par le soutien des entreprises et travailleurs turcs (Korkut, 2010).

\section{CONCLUSION}

Pour une multitude de raisons, politiques, économiques, identitaires, sécuritaires, aucun État moderne ne peut rester indifférent face au phénomène migratoire, qu'il s'agisse d'immigration et d'émigration, d'entrée sur son territoire de citoyens étrangers ou de sortie de ses propres citoyens vers un autre pays. Source d'instabilité, mais aussi de création de richesse (apport de devises) et d'instrumentalisation politique (création de groupe de pression dans le pays d'expatriation), le fait migratoire a souvent fait l'objet de politiques publiques dans plusieurs pays du monde. Ainsi la Turquie, comme d'autres, veille scrupuleusement à la nature de ses relations avec ses ressortissants, d'autant plus qu'elle a une certaine expérience en la matière puisque depuis les années 1960, des millions de

12 Voir par exemple le site de l'Union des Entrepreneurs de Turquie (Türkiye Müteahhitler Birliği), http://www.tmb.org. Les entreprises turques du secteur ont pris une grande part de la rénovation d'Almaty, de la construction de la nouvelle capitale kazakhstanaise, Astana, et se sont spécialisées dans les infrastructures aéroportuaires et routières, touristiques (grands hôtels), sanitaires (hôpitaux), éducatives (établissements secondaires, universités), industrielles. Nombreuses informations disponibles sur les sites ministériels, diplomatiques, économiques, turcs, mais souvent redondants. 
travailleurs turcs ont immigré en Europe, Allemagne, France et autres pays où la communauté turque a un poids politico-religieux qui ne peut la laisser indifférente.

En Asie centrale et dans le Caucase, elle veille plus particulièrement à ce que la présence turque ne détériore pas ses relations avec les États de la région. Dans certains cas, elle cherche même, comme d'autres États le font ailleurs, à utiliser ses ressortissants, engagés voire embrigadés dans les actions d'associations ou mouvements informels privés, pour les mettre au service de sa politique extérieure.

S'agissant des rapports entre l'État et les mouvements missionnaires turcs en Asie centrale et dans le Caucase, la République turque, séculière et laïque maintient une attitude pour le moins pragmatique. Tandis qu'en Turquie même les relations entre l'État et les organismes religieux peuvent être tendues, en Asie centrale, pour des raisons de convergence d'intérêts supérieurs, les relations sont bonnes et l'on observe même une certaine complémentarité à l'œuvre entre l'action de l'État et celles des mouvements religieux privés. Dans des pays où la société civile et les acteurs non étatiques restent pour le moins inexistants, les régimes éprouvent souvent le besoin de demander la garantie, la caution de l'État turc et de ses ambassades pour entamer un quelconque projet de coopération avec ces organisations turques. Le cas le plus représentatif est l'exemple de Fethullah Gülen et de son vaste réseau éducatif. Malgré les mauvaises relations que le mouvement entretient avec l'État en Turquie, les ambassades turques en Asie Centrale ont toujours apporté leurs soutiens aux disciples de Gülen qui ont ainsi pu plus facilement asseoir un vaste réseau d'écoles privées appuyées par des entreprises turques, elles-mêmes gérées par des expatriés turcs aux motivations religieuses affirmées.

L'implantation des mouvements islamistes turcs en Asie centrale par le biais des migrants pose la question du caractère provisoire ou définitif de cette implantation, des idées et des hommes. La plupart des migrants turcs en Asie centrale resteront sans doute dans ces pays d'accueil, car on voit déjà se multiplier les accessions à la propriété, mais surtout les mariages mixtes qui sont le meilleur indicateur du caractère définitif d'une immigration. Pour ce qui est de la prégnance des idées religieuses importées de Turquie, il convient d'être plus nuancé. En effet, leur enracinement en Asie centrale ne pourrait se faire sans concession aux pratiques et coutumes locales. Ainsi, une sorte de syncrétisme musulman turc et centrasiatique, très proches au demeurant, s'élabore chaque jour, au fur et à mesure que le temps passe et que les immigrés anatoliens prennent leurs marques dans les pays d'origine de leurs illustres aïeuls.

Quant au rêve de grandeur de la Turquie et son relent d'empire face à l'immigration turque en Asie centrale, il s'agit là d'une question complexe, dont la réponse ne peut être que partielle et temporaire. La présence des entreprises, écoles, mouvements religieux, mais aussi simples travailleurs turcs en Asie centrale a incontestablement accompagné et même aidé la Turquie à asseoir une certaine influence en Asie centrale. Toutefois cet apport ne doit pas être exagéré, car en réalité après avoir beaucoup misé sur l'Asie centrale et les retrouvailles avec les frères turcs, la Turquie est revenue à une politique plus réaliste, recentrée sur les vrais enjeux géopolitiques et stratégiques extérieurs, parmi lesquels l'Asie centrale ne figure pas. L'aventure centrasiatique, pétrie des illusions de retour dans la patrie originelle et mythique des Turcs, aura fait long feu et démontre que 
la Turquie regarde droit devant elle, vers l'Europe, et se détourne de son passé asiatique. Farouchement euro-centrée, c'est sur les millions d'immigrés turcs citoyens de l'Union européenne que la Turquie semble s'appuyer pour forcer la forteresse européenne où se concentrent ses intérêts les plus vitaux.

\section{Références bibliographiques}

ATEŞ Toktamış, KARAKAŞ Eser, ORTAYLI İlber (Dir.), Barış Köprüleri. Dünyaya Açılan Türk Okullar [Les ponts de la paix. Les écoles turques ouvertes sur le monde], Istanbul, DA (Ufuk Kitap 60, Güncel Kitaplar Dizisi 8).

BALCI Bayram (1999) Turcs et Turkmènes : les retrouvailles. Carnet d'une mission au Turkménistan, Revue Française de Géoéconomie, 10, pp. 165-175.

BALCI Bayram (2001) Hoca Ahmet Yesevi : politiques identitaires de la Turquie autour d'un cheikh centrasiatique du XIe siècle, Asien Afrika Lateinamerika, 29, pp. 91-101.

BALCI Bayram (2003a) Missionnaires de l'islam en Asie Centrale. Les écoles turques de Fethullah Gülen, Paris, Maisonneuve \& Larose, Istanbul, IFEA (Passé ottoman, présent turc).

Bayram BALCI (2003b) Les Ouzbeks d'Arabie saoudite entre intégration et renouveau identitaire via le pèlerinage, Central Asian Survey, 22 (1), pp. 23-44.

BALCI Bayram (2005) Les relations entre la Turquie et l'Asie centrale turcophone, 1991-2004, Outre-Terre, Revue Française de Géopolitique (Turquie-Europe Express, 2014 ? [La question de Chypre]), 10, pp. 297-316.

BALCI Bayram (2003c) Fethullah Gülen's missionnary schools in Central Asia: education, proselytism and Turkish Foreign Policy, Religion, State and Society, Oxford, June 03, pp. 151-177.

BALCI Bayram (2003d) Fethullah Gülen's Missionary Schools in Central Asia and their Role in the Sprading of Turkism and Islam, Religion, State \& Society, 31 (2), pp. 151-177.

BALCI Bayram (2004a) Between Sunnism and Shiism: Islam in post-Soviet Azerbaijan, Central Asian Survey, 23 (2), pp. 205-217.

BALCI Bayram (2004b) Islam et Éducation islamique en Azerbaïdjan indépendant. Premiers résultats d'une recherche en cours, Religioscope, $18 \mathrm{p}$. , http://religion.info/french/articles/ article 54.shtml.

BALCI Bayram (2008) Education, Nationalism, and Hidden Da'wa: Turkish Missionary Movements in Central Asia and the Caucasus, in Proselytization Revisited: Right Talks, Free Markets and Culture Wars, London, Equinox, pp. 365-387.

BALCI Bayram et BUCHWALTER Bertrand (collab.) (2001) La Turquie en Asie Centrale. La conversion au réalisme (1991-2000), Istanbul, IFEA, les Dossiers de l'IFEA (La Turquie d'aujourd'hui), 5.

BALCI Bayram et MOTIKA Raoul (Éds.) (2007) Religion et politique dans le Caucase postsoviétique (Les traditions réinventées à l'épreuve des influences extérieures), Paris, Maisonneuve \& Larose, Istanbul, IFEA.

BAZIN Louis (1986) Les peuples turcophones d'Eurasie : un cas majeur d'expansion linguistique, Hérodote (spécial Géopolitique des Langues), 46, pp. 75-109.

BEZANIS Lowell (1994) Soviet Muslim Emigres in the Republic of Turkey, Central Asian Survey, 13 (1), pp. 59-180.

BOZAY Kemal (2005) “... Ich bin stolz, Turke zu sein!”. Ethnisierung gesellschaftlicher Konflikte im Zeichen der Globalisierung, Schwalbach.

COPEAUX Étienne (1992) Les «Turcs de l'extérieur » dans Türkiye : un aspect du discours nationaliste turc, CEMOTI, 14, pp. 31-52.

COPEAUX Étienne (1992) Le rêve du Loup Gris, Hérodote, 64, pp. 183-194. 
COPEAUX Étienne (1994) Manuels scolaires et géographie historique : le cas turc, Hérodote, 74-75, pp. 196-240.

COPEAUX Étienne (1997) Espace et temps de la nation turque. Analyse d'une historiographie nationaliste. 1931-1993, Paris, CNRS Éditions.

COPEAUX Étienne (1997) Les territoires de référence des discours identitaires turcs, in J. Bonnemaison, L. Cambrézy et L. Quinty-Bourgeois Dir., Le territoire, lien ou frontière ? Identités, conflits ethniques, enjeux et recompositions territoriales. Actes du Colloque de la Sorbonne (2-4.10.1995), Bondy, Éditions de l'ORSTOM (Colloques et CD ROM), 9 p.

COPEAUX Étienne (2000) Une vision turque du monde à travers les cartes de 1931 à nos jours, Paris, CNRS Éditions.

ÇAĞATAY Ergun (Photographies), KUBAN Doğan (Textes) (2006) The Turkic Speaking Peoples: 2000 Years of Art and Culture from Inner Asia to the Balkans, Munich, Prestel Verlag \& The Hague, Prince Claus Fund Library.

DEMIR Emre (2007) The Emergence of a Neo-Communitarian Movement in the Turkish Diaspora in Europe: Settlement and Competition Strategies of the Gülen Movement in France and in Germany, in Muslim Words in Transition: Contribution of the Gulen Movement, London, House of Lords, SOAS, London School of Economics, http://www.gulenconference.net/index.php/ library/articles.

DIYYIH (Dış İlişkiler ve Yurtdışı İşçiler Hizmetleri Genel Müdürlüğü) (2000-2010), Yllı Raporu. Yurtdışındaki Vatandaşlarımıza İlişkin Gelişmeler ve Sayısal Bilgiler, Ankara, TC Çalışma ve Sosyal Güvenlik Bakanlığı, Annuel [voir en particulier les dernières livraisons des années 2005 et 2007 parues en 2006 et 2010 qui intègrent des chapitres Azerbaïdjan, Kazakhstan et pour la dernière Ouzbékistan].

FENOT Anne et GINTRAC Cécile (2005) Achgabad, une capitale ostentatoire. Urbanisme et autoritarisme au Turkménistan, Paris, L'Harmattan-IFEAC.

GÜLER Müjdat (2007) Orta Asya ve Kafkaslara Türk Bakışı [Vision turque de l'Asie centrale et du Caucase], Istanbul, İlgi Kültür Sanat Yay. (İnceleme Araştırma), 34 (14).

JOHANSON Lars (2006) The Borders of Turcia: Connections and Divisions in the Development of the Turkic Peoples, in E. Cagatay and D. Kuban Eds., The Turkic Speaking Peoples: 2000 Years of Art and Culture from Inner Asia to the Balkans, Munich, Prestel Verlag \& The Hague, Prince Claus Fund Library, pp. 18-29.

KORKUT Şenol (2010) The Diyanet of Turkey and Its Activities in Eurasia after the Cold War, Acta Slavica Iaponica, Hokkaido University International Symposium (The South Ossetian Conflict and Trans-border Politics in the Black Sea Rim), Tome 28, pp. 117-139.

LANDAU Jacob M. (1981), Pan-Turkism in Turkey. A Study in Irredentism, London, C. Hurst \& Co.

LANDAU Jacob M. (1995a) Pan Turkism: From Irredentism to Cooperation, Indiana University Press.

LANDAU Jacob M. (1995b) Ethnonationalism and Pan-nationalism in Turkey and the Ex-Soviet Republics, Migration, 28, pp. 67-84.

LARUELLE Marlène et PEYROUSE Sébastien (2006) Asie centrale, la dérive autoritaire (Cinq républiques entre héritage soviétique, dictature et islam), Autrement (CERI).

PAHLAVI Pierre Cyril Teymour (2002) La diplomatie culturelle à l'ère de l'interdépendance globale. La Turquie à la recherche des éléments fédérateurs de l'identité panturque, Études Internationales, 33 (2), pp. 247-274, http://www.diploweb.com/asie/pahlavil.htm.

PARLAK Nukrettin (2007) Orta Asya-Kafkasya-Balkan Ülkeleriyle İlişkiler ve Türk Dış Yardımları (1992-2003) [Les Relations et les Aides extérieures turques avec les pays d'Asie Centrale, du Caucase et des Balkans (1992-2003)], Ankara, TIKA, 91.

POUJOL Catherine (2005) Ouzbékistan. La croisée des chemins, Paris, Belin-La Documentation Française (Asie Plurielle).

SALK Gundula (1996) Die türksprachigen Afghanistanflüchtlinge in der Türkei, Materiala Turcica, 17, pp. 67-76. 
SVANBERG Ingvar (1989a) Kazak Refugees in Turkey. A Study of Cultural Persistence and Social Change, Acta Universitatis Upsaliensis, 8.

SVANBERG Ingvar (1989b) Turkistani Refugees, in P.-A. Andrews Dir., Ethnic Groups in the Republic of Turkey, Dr. Ludwig Reichert Verlag, Wiesbaden, pp. 591-601.

TAPIA Stéphane de (1998) Les réfugiés dans la construction de l'État-nation turc, Autrepart, 7, pp. 5-21.

TAPIA Stéphane de (2008) Le nouvel oléoduc Bakou-Tbilissi-Ceyhan : quels impacts pour la Turquie et l'Union européenne ?, Café géographique, in La planète en mal d'énergies ; $18^{\text {ème }}$ Festival International de Géographie de Saint-Dié des Vosges, 2007, http://www.cafe-geo.net/ article.php3?id_article=1229 ; http://fig-st-die.education.fr/actes/actes_2007/de_tapia/article. pdf.

TÜRK Fahri (2009) Azerbaycan ve Orta Asya'da Değişim Sürecinde Ortaya Çıkan Turancı Siyasi Hareketler (1989-2007) [Les mouvements pantouraniens apparus dans la période de transition en Azerbaïdjan et en Asie centrale (1989-2007)], Bilig, Türk Dünyası Sosyal Bilimler Dergisi, 51, pp. 205-230.

YAZGAN Turan (2003) Türkiye'nin Türk Dünyasındaki Eğitim-Öğretim Faaliyetleri [Les activités éducatives de la Turquie dans le Monde turc], Кати-IS, 8 (2), pp. 2-21.

YUNUSOV Arif (2009) Migration Processes in Azerbaijan, Bakou, Institute of Peace and Demography, Adiloğlu. 


\title{
Mouvements migratoires entre la Turquie et les Républiques turcophones du Caucase et d'Asie centrale : les impacts religieux
}

\author{
Bayram BALCI et Stéphane de TAPIA
}

Turquie, Azerbaïdjan et nouveaux pays indépendants d'Asie centrale partagent nombre de traits culturels, à commencer par l'appartenance au même groupe linguistique et, majoritairement, à l'islam Sunnite (ou Chiite dans le cas azerbaïdjanais). Cependant leurs histoires politiques ont largement divergé, et ce bien avant la mainmise russe puis soviétique sur les vastes territoires d'Asie centrale. Le retour à l'indépendance a autorisé de grands espoirs en Turquie, espoirs relevant aussi bien de la géopolitique que de l'économie et de la sphère linguistique et culturelle. De très nombreux accords bilatéraux ont été signés et l'Asie centrale turcophone a attiré de nombreux migrants turcs pour des raisons diversifiées. La présente contribution s'attache à l'examen d'un aspect moins connu, mais tout aussi important, celui de l'investissement religieux turc dans les territoires nouvellement indépendants, avec ses succès et ses déceptions, le tout sur fond de laïcité partagée théoriquement par la Turquie laïque kémaliste et les républiques centrasiatiques, soviétiques il y a encore peu de temps.

\section{Migrations between Turkey and Turkic-speaking Republics in Caucasus and Central Asia: Religious Impacts}

\author{
Bayram BALCI and Stéphane de TAPIA
}

Turkey, Azerbaijan and new independent countries of Central Asia share a lot of cultural elements, as belonging to the same linguistic family and, for a great majority, to Sunnî Islam, or in the case of Azerbaijan, to Shiî Islam. Nevertheless, their political histories have broadly diverged, since a long time, even before Russian and Soviet eras and political control on their territories. Return to political independence and sovereignty has authorised very great hopes and expectancies in Turkey, in various domains as geopolitics, economics, linguistic and cultural topics. A very great amount of bilateral agreements have been signed and Turkic speaking Central Asia has drained numerous Turkish migrants for diversified reasons. The present paper wants to examine a less studied, but very important, aspect which are those of the Turkish religious investments in these newly independent countries, with their successes and deceptions, even if Kemalist and secularist Turkey officially shares the same ideal of secularism with all these Central Asian republics which were members of Soviet Union, a few time ago. 


\title{
Movimientos migratorios entre Turquía y las Repúblicas de lengua turca en el Cáucaso y en Asia central: los impactos religiosos
}

\author{
Bayram BALCI y Stéphane de TAPIA
}

Turquía, Azerbaiyán y nuevos países independientes de Asia central comparten numerosos rasgos culturales, empezando por su pertenencia al mismo grupo lingüístico y, en su mayor parte, al islam Sunnita (o Chiita en el caso azerbaiyano). Sus historias políticas, sin embargo, han discrepado mucho, aun antes el dominio ruso y luego soviético en los amplios terrenos de Asia central. La vuelta a la independencia ha generado grandes esperanzas en Turquía tanto con respecto a la geopolítica como a la economía y a la esfera lingüística y cultural. Se han firmado numerosos acuerdos bilaterales y el Asia central de lengua turca ha atraído un número importante de migrantes por diversas razones. La presente contribución se dedica al examen de un aspecto menos conocido aunque igualmente importante: la implicación religiosa turca en los territorios recién independientes, con sus éxitos y sus desilusiones, teniendo en cuenta la laicidad teóricamente compartida por la Turquía laica kemalista y las repúblicas centroasiáticas que solo se deshicieron del sovietismo hace poco. 